\title{
Saturn's aurora in the January 2004 events
}

\author{
E. S. Belenkaya ${ }^{1}$, S. W. H. Cowley ${ }^{2}$, and I. I. Alexeev ${ }^{1}$ \\ ${ }^{1}$ Skobeltsyn Institute of Nuclear Physics, Moscow State University, Moscow 119992, Russia \\ ${ }^{2}$ Department of Physics and Astronomy, University of Leicester, Leicester LE1 7RH, UK
}

Received: 10 November 2005 - Revised: 12 March 2006 - Accepted: 16 March 2006 - Published: 3 July 2006

\begin{abstract}
Differences in the solar wind interaction with the magnetosphere of Saturn relative to the Earth result from the decrease in the solar wind plasma density and magnetic field strength with distance from the Sun, and from the change in the average angle of the IMF at Saturn's orbit. Other reasons are related to Saturn's rapid rotation and internal magnetospheric plasma sources. Moreover, the IMF structure observed by Cassini in 2003-2004 during the approach to Saturn is consistent with corotating interaction regions (CIRs) existing during the declining phase of the solar cycle. Two cases on 16 and 26 January 2004 are considered when disturbances in the solar wind passed Cassini and then Saturn. After the solar wind shock encountered the kronian magnetosphere, the auroral oval became brighter (especially at dawn) with a reduced radius. In these cases the auroral power was anti-correlated with the radius of the oval. Possible mechanisms responsible for such unexpected behavior are presented and discussed in detail.
\end{abstract}

Keywords. Magnetospheric physics (Auroral phenomena; Planetary magnetospheres; Solar wind-magnetosphere interactions)

\section{Introduction}

Saturn is a rapidly rotating planet with an angular velocity of $\Omega_{S}=1.638 \cdot 10^{-4} \mathrm{~s}^{-1}$, possessing a strong magnetic field. The dipole magnetic moment of Saturn, $\mathbf{M}_{S}$, is equal to 4.6.10 $10^{13} \mathrm{G} \cdot \mathrm{km}^{3}$ or $0.2 \mathrm{G} \cdot R_{S}{ }^{3}$ and is directed to the north, opposite to that of the Earth but the same as Jupiter's field. The conventional Saturn radius is $R_{S}=60330 \mathrm{~km}$.

The subsolar magnetopause distance of the kronian magnetosphere is $R_{S s} \sim 17-24 R_{S}$ (Behannon et al., 1983; Maurice and Engel, 1995). Plasma motions in Saturn's magnetosphere are driven by planetary rotation and the solar wind

Correspondence to: E. S. Belenkaya

(elena@dec1.sinp.msu.ru) interaction (e.g. Cowley et al., 2004a). As noted in the work of Smith et al. (1980), the corotation electric field can dominate the convective electric field due to the solar wind out to radial distances in excess of $21 R_{S}$ (the average radial position of the noon magnetopause boundary). The plasmas in the kronian magnetosphere rigidly corotate roughly out to $10 R_{S}$. The configuration and dynamics of the Saturnian magnetosphere, as well as the ionospheric structure and morphology of auroral emissions have been reviewed in detail by Blanc et al. (2002).

Cowley and Bunce (2003) noted that the observed auroras at Saturn occur closer to the pole (at $\sim 10^{\circ}-15^{\circ}$ co-latitude) than in the case of Jupiter $\left(\sim 16^{\circ}\right)$. The Hubble Space Telescope (HST) data presented by Cowley et al. (2004a) indicate the latitudinal width of Saturn's auroral oval to be typically $\sim 0.3^{\circ}-0.6^{\circ}$. The poleward boundary of Saturn's UV aurora thus lies close to the expected boundary of open and closed field lines (Badman et al., 2005). The main oval auroras also often present a spiral aspect, such that in the dusk sector two arcs co-exist simultaneously with a longitudinal overlap of $\sim 2 \mathrm{~h}$ of local time (Gérard et al., 2004). Cowley and Bunce (2003) argued that the main oval emissions at Saturn, unlike those at Jupiter, are not associated with the corotation-enforced current system, but are connected with solar wind-magnetosphere coupling currents, as at Earth. Clarke et al. (2005) mentioned that Saturn's auroral emissions vary slowly; some features appear in partial corotation whereas others are fixed in local time, thus possibly relating to the solar wind. Gerard et al. (2004) stated that the main ovals are co-located, with the narrow upward field-aligned current system associated with the departure from plasma corotation near the open-closed field line boundary. Crary et al. (2005) and Bunce et al. (2006) showed that Saturn's aurora strongly echoes the structuring of the solar wind and its magnetic field.

A comparison of the terrestrial and kronian auroras is given, for example, by Prangé et al. (2004). At Earth, the quiet aurora is located on closed magnetotail field lines.

Published by Copernicus GmbH on behalf of the European Geosciences Union. 


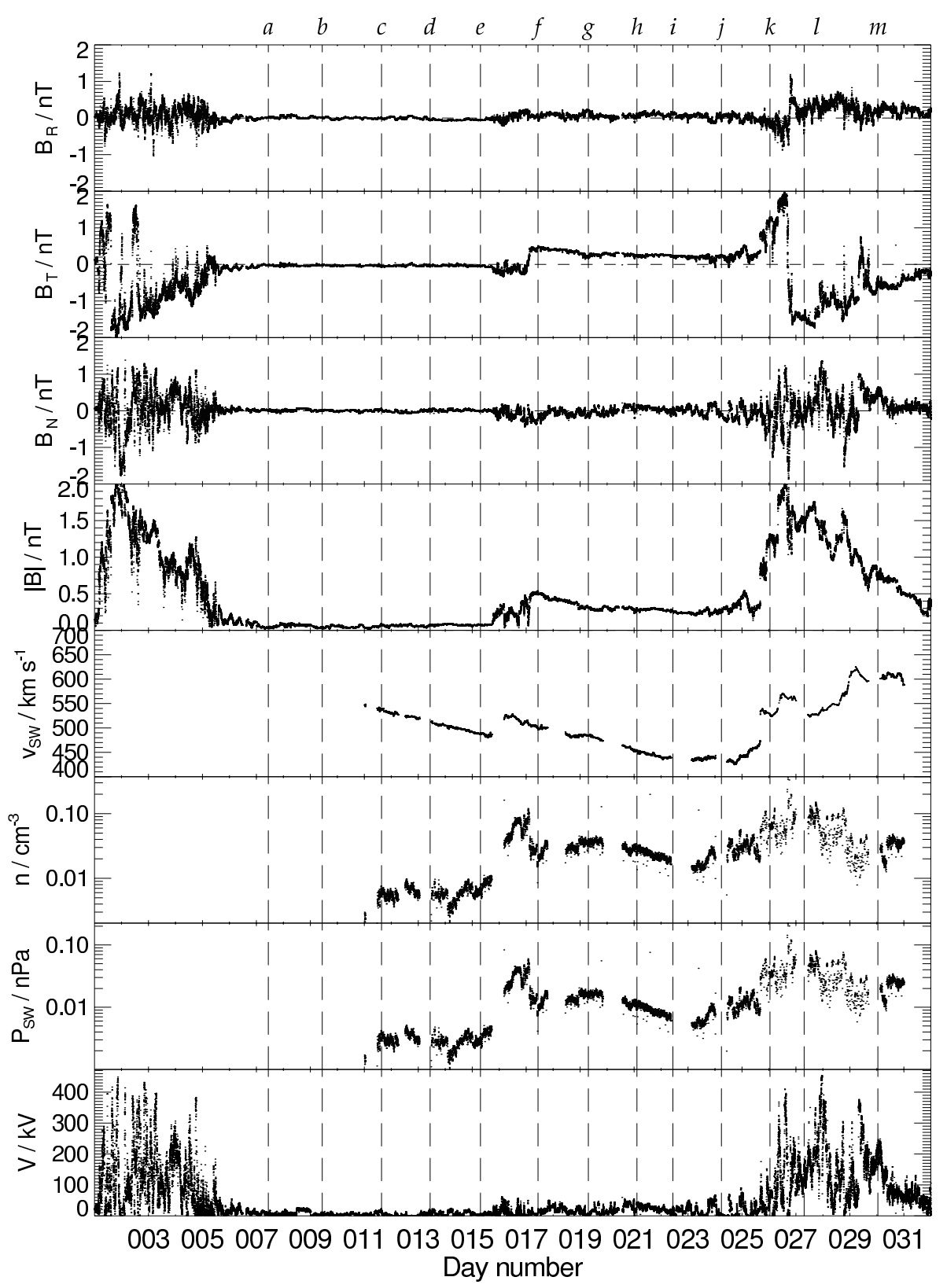

Fig. 1. Stack plot of Cassini magnetic field and plasma data. The first four panels show the RTN magnetic field components $\left(B_{R}, B_{T}, B_{N}\right)$ and magnetic field $|B|$ in nT. The fifth, sixth, and seventh panels show the solar wind proton density $n_{p}$, the solar wind velocity $v$, and the dynamic pressure $\left(P_{S w}\right)$, respectively. The bottom panel shows the estimated reconnection voltage $\Phi$ in $\mathrm{kV}$ (Jackman et al., 2004). The dashed vertical lines indicate the times of the HST images, shifted by $17 \mathrm{~h}$ (the estimated time for solar wind propagation from Cassini to Saturn at a mean solar wind speed of $500 \mathrm{~km} \mathrm{~s}^{-1}$ ) (Badman et al., 2005).

Compression of the magnetosphere by a shock leads to dramatic shifts towards low latitudes and brightening of the auroral oval. Increased emission at the dayside cusp can be observed as a result of reconnection. Terrestrial auroral activity is mainly under solar wind control. The normal Saturn aurora also suggests some kind of solar wind-related local time organization in the steady-state. But in contrast with the Earth, an asymmetry in latitude and brightness develops between dawn and dusk. During solar wind-related storms, the auroral latitudes are almost unchanged (or expand polewards) and the oval is significantly brighter, especially in the midnight-dawn sector.

Cowley et al. (2004a) noted that the outermost magnetospheric region driven by the interaction with the solar wind is dominated by reconnection between the southwarddirected planetary field in the equatorial magnetosphere and 


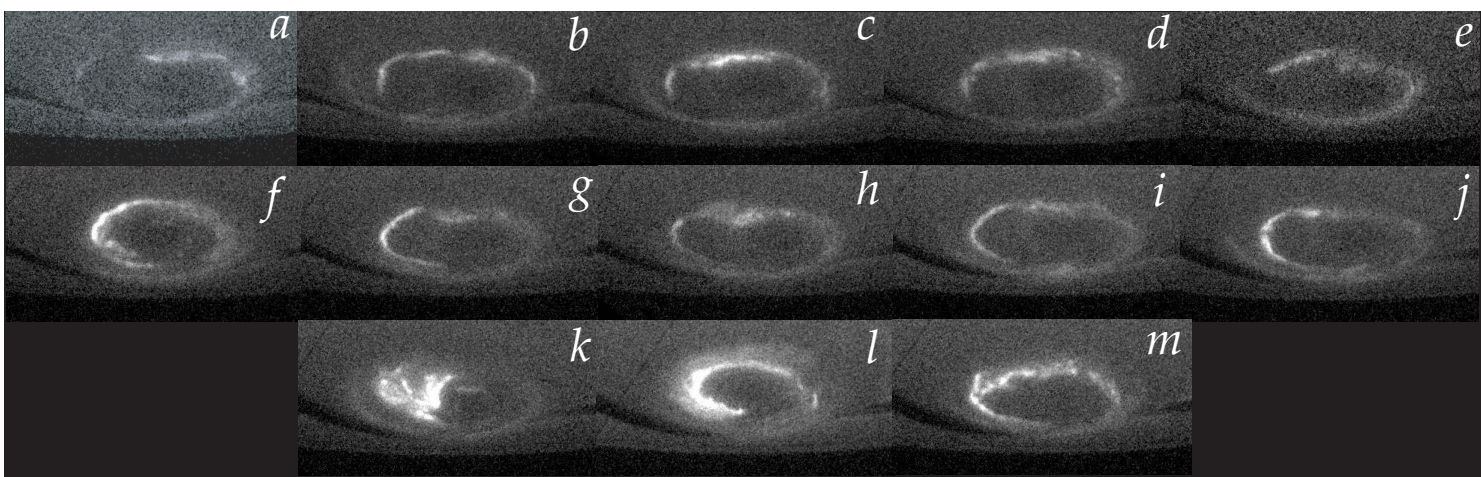

Fig. 2. Ultraviolet images of Saturn's southern aurora from HST-STIS obtained on 8, 10, 12, 14, 16, 18, 20, 21, 23, 24, 26, 28, and 30 January 2004 (panels a to $\mathbf{m}$, respectively). The panels have been generated by combining individual images obtained on a given HST orbit (Clarke et al., 2005; Bunce et al., 2006). The noon meridian in the centre top of each plot, and dawn to the left.

a northward pointing IMF. The solar wind electric field is $\mathbf{E}_{\mathrm{IEF}}=-\left[\mathbf{V}_{s w} \times \mathbf{B}_{\mathrm{IMF}}\right]$. On the average, the solar wind speed, $\mathbf{V}_{s w}$, is approximately constant with heliocentric distance, while the interplanetary magnetic field (IMF), $\mathbf{B}_{\mathrm{IMF}}$, varies inversely with distance. Thus, at Saturn the interplanetary fields $\mathbf{B}_{\mathrm{IMF}}$ and $\mathbf{E}_{\mathrm{IEF}}$ should be about 10 times weaker than at Earth. Although at the orbit of Saturn the energy density of the IMF has decreased by two orders of magnitude over its value at $1 \mathrm{AU}$, the solar wind magnetic and electric fields are significant for the kronian magnetosphere.

\section{Interaction of Saturn's magnetosphere with the com- pression regions in the solar wind}

Differences in the interaction of the solar wind with the magnetosphere of Saturn, relative to the Earth, result from a decrease in the interplanetary plasma density (from $\sim 5 \mathrm{~cm}^{-3}$ at the Earth to $\sim 0.03 \mathrm{~cm}^{-3}$ at Saturn) and magnetic field strength (from $\sim 6 \mathrm{nT}$ at the Earth to $\sim 0.3 \mathrm{nT}$ at Saturn), as well as from the change in the average angle of the IMF (Maclennan et al., 1983). Other reasons are Saturn's rapid rotation and internal magnetospheric plasma sources. Most recently, Jackman et al. (2004) examined the IMF structure observed by Cassini at the heliocentric distance of $\sim 9 \mathrm{AU}$, and noted the persistent corotating interaction regions (CIRs) existing during the declining phase of the solar cycle. In particular, Jackman et al. (2004) noted that the structuring of the interplanetary medium into regions of high and low field strength leads to corresponding temporal structuring of the interaction between the solar wind and the kronian magnetosphere. It was shown that during the compression regions that are short in duration the IMF was $\sim 0.5-2 \mathrm{nT}$, while during the longer rarefaction intervals the IMF was $\sim 0.1 \mathrm{nT}$ or less.

Here, following Bunce et al. (2006) and Badman et al. (2005), we consider the solar wind magnetometer and plasma data for January 2004 measured by Cassini, together with the UV aurora in Saturn's southern ionosphere (Clarke et al., 2005) observed simultaneously by the Hubble Space Telescope (HST) (see Figs. 1 and 2, respectively). The design and operation of the Cassini Plasma Spectrometer (CAPS) instrument that obtained the plasma data is described in detail by Young et al. (2004), while the magnetic field instrumentation is described by Dougherty et al. (2004). In general, the magnetic field was very disturbed, with quickly varying orientations. In Fig. 1 (taken from Fig. 1 of Badman et al., 2005), the RTN spherical polar system is used to show the field components in the upper panels, with $B_{r}$ directed radially outward from the Sun, $B_{t}$-azimuthal in the direction of planetary motion, and $B_{n}$ positive northward from the equatorial plane (see also Crary et al., 2005). For January 2004 the difference between the Kronian Solar-Magnetospheric (KSM) coordinate system and the RTN system was very small (the angle between the $N$ axis and Saturn's spin (and magnetic axis) projected onto the $N-T$ plane was $\leq 4^{\circ}$ ). Below we ignore this small angle, such that the KSM and RTN components are related by $B_{r}=-B_{x}, B_{t}=-B_{y}$, and $B_{n}=B_{z}$. The next three panels in Fig. 1 display the solar wind velocity $\left(v_{s w}\right)$, density $(n)$, and dynamic pressure $\left(P_{s w}\right)$. The final panel shows the kronian magnetopause reconnection voltage, estimated using the algorithm of Jackman et al. (2004).

The HST images shown in Fig. 2 were previously presented by Clarke et al. (2005). The entire southern oval is seen, owing to the $\sim 26^{\circ}$ tilt of Saturn's spin axis at this epoch. The center of the near-circular auroral oval is offset by $\sim 3-4^{\circ}$ toward midnight.

Clarke et al. (2005), Bunce et al. (2006), and Badman et al. (2005) describe two particular intervals on 16 and 26 January 2004. In both of them disturbances in the solar wind first passed Cassini and then Saturn during the period of observations, associated with CIR compressions bounded by a forward shock. After the solar wind shock encountered the kronian magnetosphere, the auroral oval became brighter (especially at dawn) with a reduced radius. In these cases 
the auroral power was anti-correlated with the radius of the auroral oval. Considering these events, Clarke et al. (2005) noted that the behavior of Saturn's aurora in response to a large solar wind dynamic pressure increase is different from those of the terrestrial and Jovian systems. The phasing of the HST observations were such that the increased auroral brightness was observed 41 and $9 \mathrm{~h}$ after the corresponding solar wind shocks had arrived at the bow shock of Saturn's magnetosphere for the 16 and 26 January 2004 cases, respectively. The latter large disturbance led to the strongest auroral brightenings on 26-28 January, when the auroral power increased by a factor of three from the previous mean level. The dawn side polar cap was completely filled with bright Sun-aligned, arc-like emissions on 26 January. Let us consider these two cases in more detail.

On 16-17 January, after an 8-day rarefaction interval, a "minor" compression region was observed, with modest increases in magnetic field magnitude (from $0.1 \mathrm{nT}$ to $0.5 \mathrm{nT}$ ), density (from $0.006 \mathrm{~cm}^{-3}$ to $0.03 \mathrm{~cm}^{-3}$, and then to $0.1 \mathrm{~cm}^{-3}$ ), and flow speed (from $480 \mathrm{~km} \mathrm{~s}^{-1}$ to $530 \mathrm{~km} \mathrm{~s}^{-1}$ ). After the shock arrival, $B_{x}$ remained near zero, $B_{y}$ changed from slightly positive to slightly negative $(\sim-0.4 \mathrm{nT})$, and $B_{z}$ turned to predominantly southward $(-0.4 \mathrm{nT})$ from the near zero level (in the Solar-Magnetospheric Saturnian coordinates).

The simultaneous HST data showed a quiet auroral oval throughout the rarefaction intervals. After the shock arrival, associated with the beginning of the minor compression region (16-17 January), the oval contracted and became much brighter. Bunce et al. (2006) explained these features by the onset of strong tail reconnection and the closure of the open flux in the tail, which should occur in response to the forward shock waves associated with CIR compression regions. After contraction, the oval slowly increased in area, which Bunce et al. (2006) connected with an increase in dayside reconnection and the accumulation of open flux in the tail.

On 25-31 January the "major" compression region occurred. At the shock the field strength jumped from $0.3 \mathrm{nT}$ to $1 \mathrm{nT}$, with peaks of $2 \mathrm{nT}$; the velocity increased from $470 \mathrm{~km} \mathrm{~s}^{-1}$ to $540 \mathrm{~km} \mathrm{~s}^{-1}$ (with a subsequent peak of $\sim 620 \mathrm{~km} \mathrm{~s}^{-1}$ ), and the density grew from $0.03 \mathrm{~cm}^{-3}$ to $0.1 \mathrm{~cm}^{-3} . B_{x}$ turned from a near-zero level to positive values $\sim 0.5 \mathrm{nT}$, while $B_{y}$ became negative $(\sim-2 \mathrm{nT})$, and $B_{z}$ became southward $(\sim-1.4 \mathrm{nT})$ and shortly after that predominantly northward.

After the sudden, strong increase in field strength and solar wind velocity in the 26 January event, the oval also contracted and became brighter at the dawn. Moreover, the polar cap dawn side was then filled with bright, arc-like emissions. Bunce et al. (2006) and Cowley et al. (2005a) suggested that it is connected with the onset of tail reconnection triggered by the sudden compression of the magnetosphere. Cowley et al. (2005a) discussed how this scenario leads to a strong correlation of auroral disturbances at Saturn with the solar wind dynamic pressure rather than to a correlation with the north-south IMF component, as observed at Earth. Jackman et al. (2004) and Bunce et al. (2006) estimated the voltage across Saturn's magnetopause associated with the production of open flux, to be $\leq 10 \mathrm{kV}$ for the rarefaction regions, $\sim 35 \mathrm{kV}$ with peaks of $\sim 100 \mathrm{kV}$ for the 16-17 January event, and $\sim 150 \mathrm{kV}$ peaking at $\sim 500 \mathrm{kV}$ for the 25-31 January event.

Clarke et al. (2005) assumed the possibility of different states of Saturn's magnetosphere, leading to different auroral emission distributions. Such states could be related to the orientation of Saturn with respect to, or variations in, the solar wind and IMF.

Here we will briefly discuss the reaction of the magnetosphere to the encounter with the compression region in the solar wind. Tamao (1975) constructed a conceptual model for unsteady interactions of solar wind disturbances with the Earth's magnetosphere. Wilken et al. (1982) considered the processes arising in the terrestrial magnetosphere in response to the impulsive changes in solar wind parameters, using simultaneous spacecraft and ground-based observations. They showed that isotropic compressional hydromagnetic waves are generated all along the magnetopause during the dynamical compression of the magnetosphere. This fast-mode wave propagates inward with a velocity somewhat higher than the local Alfvén velocity. The polarisation current $i_{p}$ in the wave front accelerates plasma to the new conditions. In this sense the step-like waves act as switch-on waves. In addition to the inward propagation process across the field lines, Alfvén waves are also generated which propagate along the field towards the ionosphere (Tamao, 1975). These waves couple the physical processes in the magnetosphere to the ionosphere, switching on a transient field-aligned current system $j_{\|}$.

The Alfvén velocity in the Earth's outer dayside magnetosphere, $1000-1300 \mathrm{~km} \mathrm{~s}^{-1}$ (Wilken et al., 1982), exceeds the speed of the "front" in the solar wind propagating along the magnetopause $\left(\sim 400 \mathrm{~km} \mathrm{~s}^{-1}\right)$. Thus, we may conclude that all phenomena in the terrestrial magnetosphere connected with the waves arise faster than those associated with the changing solar wind parameters at the front. In particular, the tailward-travelling disturbance transmitted by the waves spreads faster than the magnetospheric effects produced by the changing IMF strength and direction across the interplanetary shock. So first, we should consider what will happen due to the wave propagation through the Earth's magnetosphere and after that, how the IMF rotation will manifest itself in magnetosphere-ionosphere coupling.

Let us consider what happens in Saturn's magnetosphere during its encounter with a compression region in the solar wind (however, it should be noted that in this case the current directions are opposite, as the magnetic moments of the Earth and Saturn are antiparallel to each other). An analysis of waves in Saturn's dayside magnetosphere has been undertaken by Lepping et al. (2005), using Voyager 1 data. Lepping et al. (2005) found that there are two types 


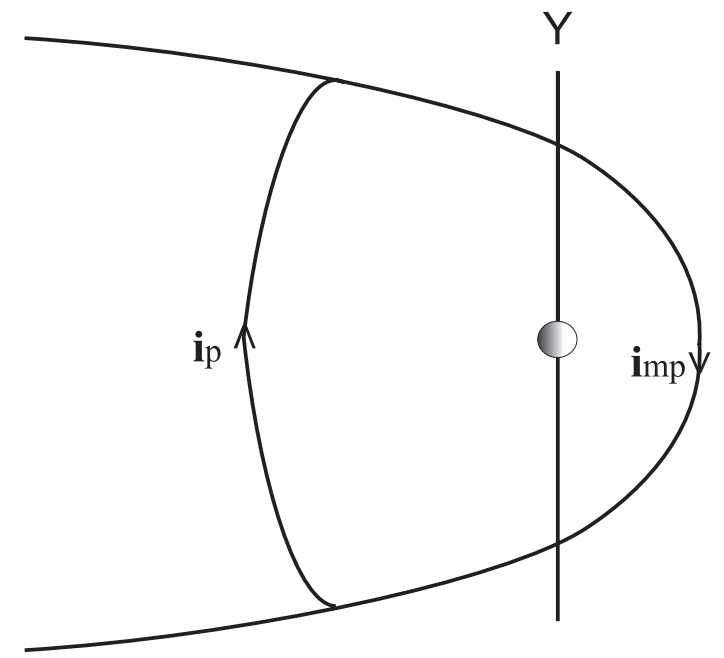

Fig. 3. Sketch of "transient ring current" in the equatorial section of the kronian magnetosphere. Polarisation current $i_{p}$ and diamagnetic current $i_{m p}$ located on the disturbed portion of the kronian magnetopause bound the compressed region as it expands tailward.

of MHD waves in Saturn's outer dayside magnetosphere: field-aligned propagating ion-cyclotron waves (mainly in the plasma sheet region away from the equator), and lowerfrequency higher-power waves which propagate/convect in the azimuthal direction predominantly in the mantle region near the equatorial plane. They also considered hydromagnetic surface waves which mode-convert to kinetic Alfvén waves. The fluctuations propagating in the azimuthal direction are convected MHD waves which have a strong compressional component and are believed to be due to the centrifugal flute instability (Lepping et al., 2005).

We suggest that the polarisation current $i_{p}$ at the wavefront of the compressed region (disturbed zone in the magnetosphere which expands tailward from the dayside kronian magnetopause), flowing from dawn to dusk (in Fig. 9 of Wilken et al., 1982, in the case of the Earth, $i_{p}$ flows from dusk to dawn), can be closed primarily by the diamagnetic current on the disturbed portion of the magnetopause $i_{m p}$. Figure 3 illustrates the current system arising in Saturn's equatorial plane (we will call it a "transient ring current"). This system resembles Fig. 1a of Tamao (1975), representing transient electric currents in the Earth's magnetosphere which appear after an encounter with the interplanetary shock at which the IMF turns southward. The "transient ring current" includes the polarisation current $i_{p}$ and an enhanced Chapman-Ferraro current $i_{m p}$ circulating around the boundary of the compressed region. The magnetic field of the "transient ring current" in the noon-midnight meridional cross section is directed southward inside the compressed region and northward outside of it (see Fig. 4A).

In the kronian tail current sheet, near its inner edge, the southward background magnetospheric magnetic field is
A

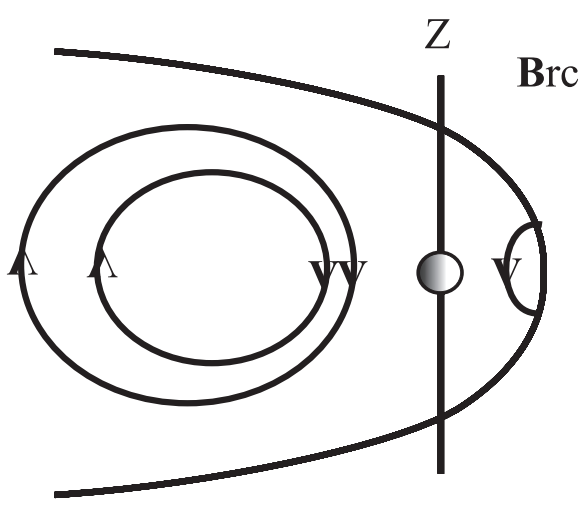

B
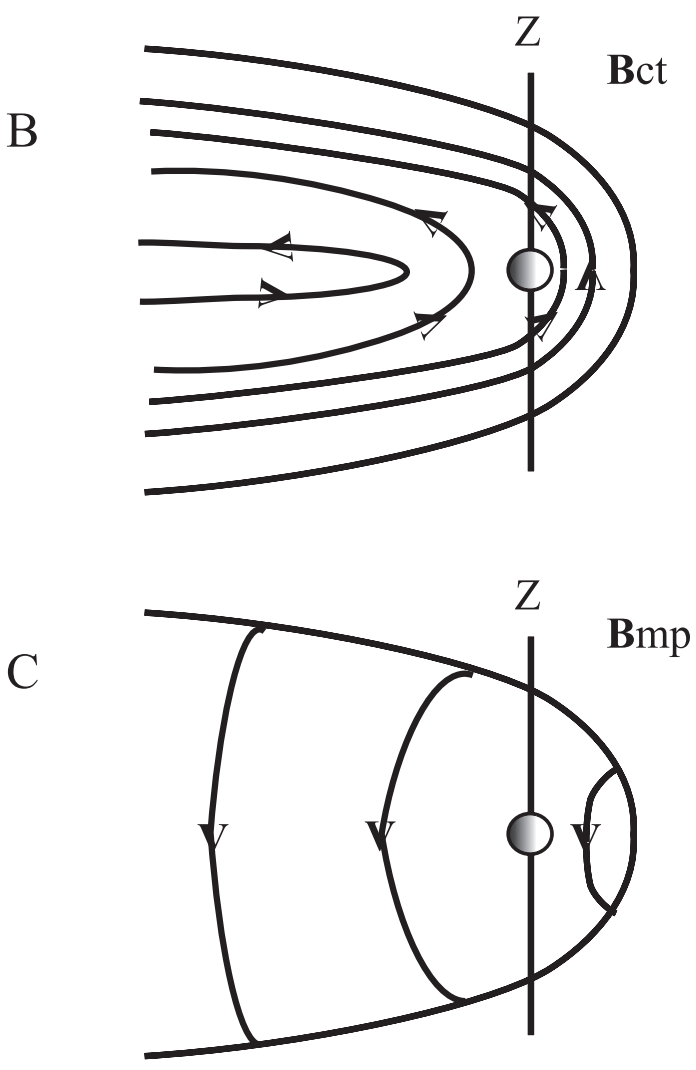

Fig. 4. Schematic diagrams of the magnetic field in a noon-midnight cross section for the following kronian current systems: (A) the "transient ring current" $\left(B_{r c}\right)$; $(\mathbf{B})$ the tail current system $\left(B_{c t}\right)$, and $(\mathbf{C})$ the magnetopause currents $\left(B_{m p}\right)$ (following Maltsev and Ostapenko, 2000, for the Earth).

mostly weakened by the northward magnetic field of the tail current system (see Fig. 4B, C). This is why this is a preferable place where the appropriate conditions for reconnection may be realized. Thus, the onset of tail reconnection triggered by the sudden compression of the magnetosphere (Bunce et al., 2006) arises. From Fig. 1 it is seen that a high pressure existed during the two events investigated (on 16 and 26 January 2004), so during these time periods the 


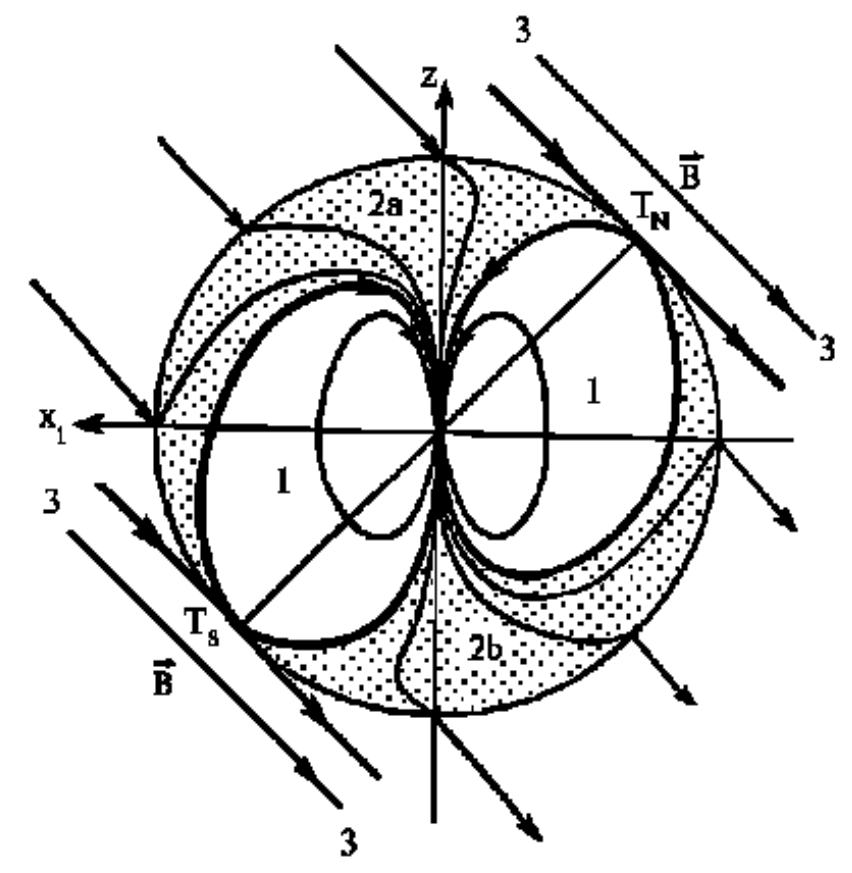

Fig. 5. Meridional section including southward IMF in the spherical terrestrial magnetospheric model (Alexeev and Belenkaya, 1983).

appropriate conditions for reconnection near the inner edge of the tail current sheet could be realized. As a consequence, the corresponding reduction in the polar cap area can be expected.

It should be noted that the creation of the "transient ring current" in Saturn's equatorial plane is executed by the fastmode wave sweeping tailward with a velocity a little higher than the local Alfvén velocity. In Saturn's magnetosphere, according to the results of Lepping et al. (2005), the Alfvén speed ranges from $156 \mathrm{~km} \mathrm{~s}^{-1}$ at $8 R_{S}$ to $52 \mathrm{~km} \mathrm{~s}^{-1}$ at $13 R_{S}$. From these data we may conclude that in the equatorial kronian magnetosphere, the Alfvén speed is less than the propagation speed of the front of the compression region in the solar wind (>500 $\mathrm{km} \mathrm{s}^{-1}$, see Fig. 1). So, while the disturbance spreads tailward in the equatorial magnetospheric plane with the fast-mode speed, the CIR front reaches the cusp regions, and other processes connected with the IMF rotation begin to develop. Both of these mechanisms can be responsible for the auroral features observed in Fig. 2. Let us now consider the second of these in more detail.

\section{Interaction between the interplanetary and kronian magnetic fields in the January 2004 events}

Different types of reconnection of the terrestrial magnetospheric magnetic field with northward IMF have been discussed in the literature (see, for example, Russell et al., 1972; Cowley, 1973; Belenkaya, 1993, and Milan, 2004). Here we will consider the reconnection processes during which

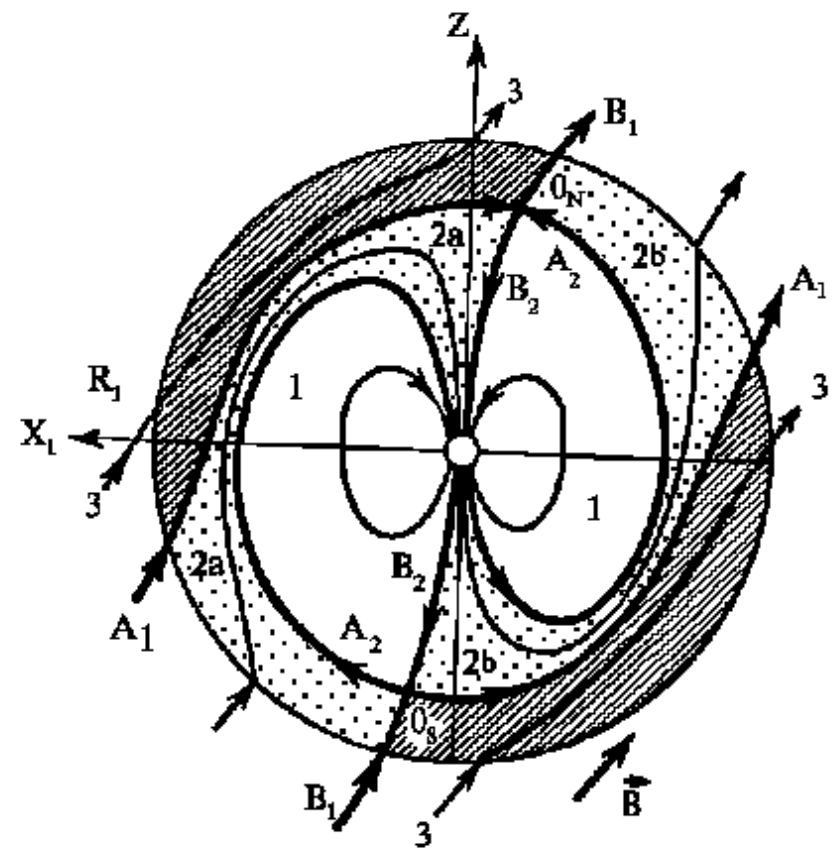

Fig. 6. Meridional section including northward IMF in the spherical terrestrial magnetospheric model (Alexeev and Belenkaya, 1983).

northward interplanetary and closed field lines form open magnetic field lines in both polar caps at one merging site, while these open field lines reconnect to produce interplanetary and closed field lines again at the other merging site (Belenkaya, 1998a, b). Figures 5 and 6 (from Alexeev and Belenkaya, 1983, based on the work of Cowley, 1973) show schematically the difference between the Earth's magnetospheric magnetic field configuration for southward (Dungeylike) and northward IMF. It is seen that in the steady state, the open field line "windows" at the magnetopause are narrower for northward IMF than for southward IMF. This effect was also demonstrated by Alexeev (1986) in his paraboloid terrestrial model (see also Belenkaya, 1998a).

For the Earth it was shown by Clauer et al. (2001) and Belenkaya et al. (2004) that after the encounter of the terrestrial magnetosphere with a solar wind dynamic pressure pulse associated with a simultaneous northward turning of the IMF, a transition current system arises. The high-latitude, threedimensional transition current system includes the $N B Z$ (in the cusp region) and the Region 1 (at the open-closed field line boundary) field-aligned currents, closed by ionospheric Pedersen currents flowing on open field lines. The fieldaligned $N B Z$ currents, which exist during intervals of northward IMF, are directed opposite to the Region I field-aligned currents. They are distributed poleward of the Region I field-aligned currents and their intensity increases towards the cusp. After the transition current system formed, the Earth's auroral oval became thick and bright, while the polar cap contracted. It was shown that despite a strong jump 
in dynamic solar wind pressure, the polar cap area decreased due to the northward IMF rotation from the near horizontal orientation.

Belenkaya et al. (2004) presented an analysis of the dependence of the intensity and direction of the field-aligned currents in the transition current system on the radial $\left(B_{x}\right)$ and azimuthal $\left(B_{y}\right)$ components of the IMF, and on the highlatitude ionospheric conductivity. The strength of the Region 1 field-aligned currents in the transition current system was calculated using the change in the ionospheric electric field components, $\left.\left\{E_{\theta}\right\}\right|_{\theta_{m}}$, normal to the open field line region boundary at the ionospheric level (this boundary is determined by the co-latitude $\theta_{m}$ ). The transition current system exists during a characteristic time period, $t_{r}$, after the terrestrial magnetosphere encounters an interplanetary shock containing a northward rotation of IMF. During this characteristic time a convection corresponding to the northward IMF is established in the polar caps, but is still absent on closed field lines. The ionospheric closure currents in the transition current system correspond to a situation where the "effective low-latitude ionospheric conductivity" is equal to zero. Thus, during the period $t_{r}$, the electric field driven by the solar wind is located only within the open field line regions. So, the value of $\left.\left\{E_{\theta}\right\}\right|_{\theta_{m}}$ is then determined only by the normal component of the electric field on the open field line side of its boundary.

We suggest that a similar situation occurred at Saturn during the two cases considered. In both of them, simultaneously with the solar wind pressure jump, the IMF turned southward for some time, which is equivalent to a northward IMF turning for the Earth. This is why, after the arrival of the interplanetary shock associated with the southward turning of IMF, the polar cap contracted, and the dawn side of the auroral oval became bright: the upward Region 1 field-aligned currents are located there for the cases under consideration. Using the method described by Belenkaya et al. (2004), we can obtain analytical expressions for the normal component of the electric field on the open field line region at its boundary, $\left.E_{n}\right|_{\theta_{m}}$ :

$\left.E_{\theta}\right|_{\theta_{m}}=\frac{\delta \Phi_{p c}}{2 R_{S} \sin \theta_{m}}\left[\sin \varphi-\frac{\cos \varphi \cot \frac{\varphi_{m}-\varphi}{2}}{\cos \theta_{m}}\right]$

and for the corresponding field-aligned current linear density at the boundary of the open field lines in the southern Saturnian polar cap

$I_{\|}=-\Sigma_{P}^{*} \frac{\delta \Phi_{p c}}{2 R_{S} \sin \theta_{m}}\left[\sin \varphi-\frac{\cos \varphi \cot \frac{\varphi_{m}-\varphi}{2}}{\cos \theta_{m}}\right]$,

where $\delta \Phi_{p c}$ is the potential drop across the polar cap. The polar cap is approximated by a circle with a radius $R_{S} \sin \theta_{m}$, where center $O^{\prime}$ is slightly shifted from the Saturnian magnetic pole. In the coordinate system $(\theta, \varphi), O^{\prime}$ is the pole, $\theta$ is the polar angle, and $\varphi$ is the azimuthal angle, measured from the noon meridian anticlockwise. Here $\Sigma_{P}^{*}$ is the effective height-integrated ionospheric Pedersen conductivity (according to the estimation of Bunce et al. (2003), it is $\sim 1-2$ mho). The effective value of the ionospheric Pedersen conductivity, $\Sigma_{P}^{*}$, is less than the true value, $\Sigma_{P}$, due to the "slippage" of the neutral atmosphere from rigid corotation (see, for example, Cowley et al., 2005b). $\varphi_{m}$ is the angle between the Saturn-Sun line (the $X$ axis) and the IMF projection onto the plane $(X, Y)$, which is determined by equations:

$\sin \varphi_{m}=B_{y} / B_{l}, \quad \cos \varphi_{m}=B_{x} / B_{l}, \quad B_{l}=\sqrt{B_{x}^{2}+B_{y}^{2}}$.

As the radius of the open field line region in the polar cap is relatively small $\left(\cos \theta_{m} \sim 1\right)$, we can obtain an approximate expression from Eq. (2) (see Alexeev and Belenkaya, 1985):

$I_{\|}=\left.\Sigma_{P}^{*}\left\{E_{\theta}\right\}\right|_{\theta_{m}} \approx-\frac{\delta \Phi_{p c}}{R_{S}} \Sigma_{P}^{*} \frac{\cos \frac{\varphi+\varphi_{m}}{2}}{2 \sin \theta_{m} \sin \frac{\varphi-\varphi_{m}}{2}}$.

It should be noted that the Region 1 field-aligned currents in the Saturnian and terrestrial magnetospheres are oppositely directed (the ordinary Region 1 field-aligned currents in the terrestrial magnetosphere are associated with southward IMF and duskward electric field in the polar caps, while the Saturnian Region 1 field-aligned currents correspond to northward IMF and dawnward electric field). By analogy, in the transition current systems of these two planets, the Region 1 field-aligned currents are also oppositely directed and correspond to northward IMF and the dawnward polar cap electric field in the terrestrial magnetosphere, and to southward IMF and the duskward electric field in the kronian magnetosphere. By analogy with the terrestrial magnetosphere, currents similar to the field-aligned currents distributed near the cusp on open field lines for northward IMF at Earth, and called $N B Z$ currents, should also exist in the Saturnian magnetosphere for southward IMF. In this case, we can discuss " $S B Z$ " currents for Saturn (terrestrial $N B Z$ and kronian $S B Z$ currents are also oppositely directed).

The directions of the Region 1 field-aligned currents in the transition current systems arising in the two events described above (16 and 26 January 2004) can be obtained from Eq. (4). The strong upward Region 1 currents generated after the arrival at the kronian magnetosphere of the interplanetary shock, accompanied by the southward turning of IMF, could be associated with the bright regions of the Saturnian auroral oval. For the case of 16-17 January $\left(B_{x}=0\right.$, $B_{y}=-0.4 \mathrm{nT}, B_{z}=-0.4 \mathrm{nT}$ ), the ionospheric projection of the southern cusp, $O_{S}^{\prime}$, was located at the dawn meridian of the southern polar cap boundary (due to the negative $B_{y}$ ). In the open field line region of the southern polar cap, one convection vortex (due to $B_{x} \approx 0$ ) existed with a singularity at $O_{S}^{\prime}$ $\left(\varphi=\varphi_{m}=3 \pi / 2\right)$. In the vicinity of the singularity, the strong upward Region 1 currents were located, while the downward Region 1 field-aligned currents were concentrated along the rest of the auroral oval. Figure 7A shows the convection 

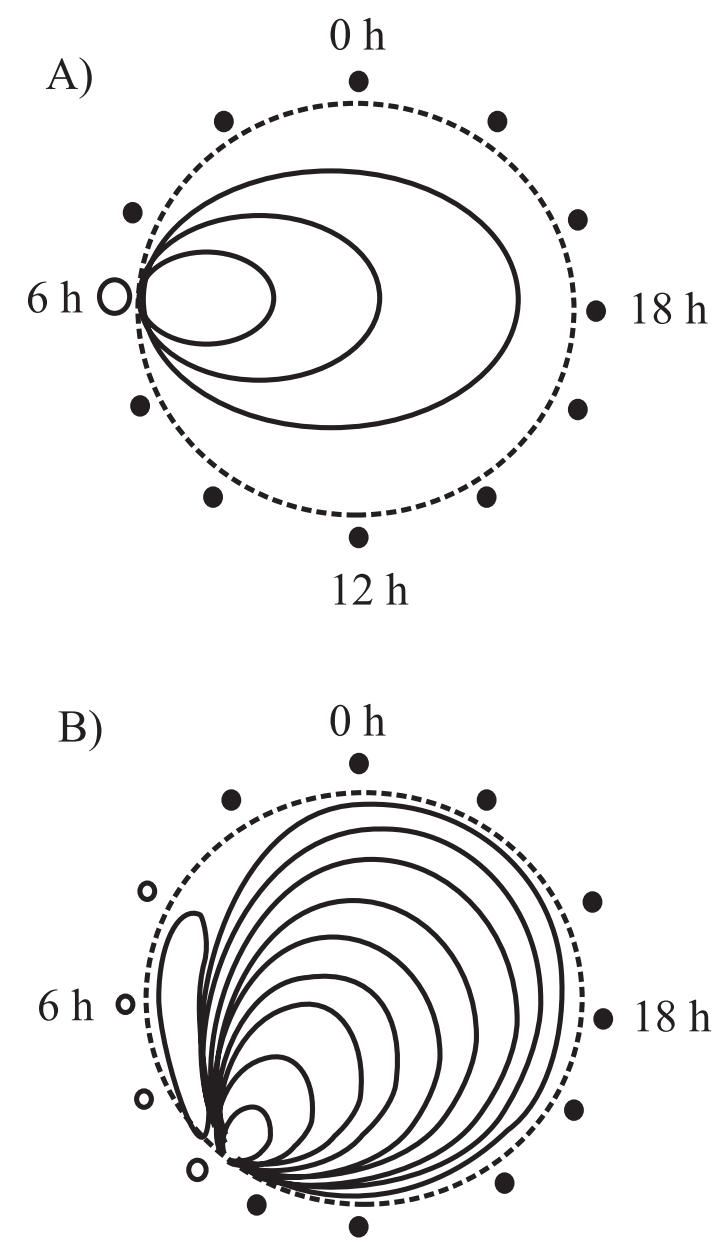

$12 \mathrm{~h}$

Fig. 7. Convection driven by the solar wind electric field and the Region 1 field-aligned currents. The diagrams show the southern Saturnian polar cap after the sudden increase in the IMF field strength and the solar wind velocity and density, simultaneously with the southward IMF turning: (A) beginning of the 16-17 January 2004 event; (B) beginning of the 25-31 January 2004 event. Open circles indicate upward currents, while filled circles indicate downward currents. The open-closed field line boundary is marked by the dashed curve. Polar cap convection features are obtained by the method described by Alexeev and Belenkaya (1985) for the terrestrial magnetosphere using the scaling of all parameters for the Saturn magnetosphere.

patterns driven by the solar wind electric field and the corresponding Region 1 currents at the beginning of the 16-17 January event (open circles indicate upward currents, while filled circles indicate downward currents).

For the case of 26 January $\left(B_{x}=0.5 \mathrm{nT}, B_{y}=-2 \mathrm{nT}\right.$, $B_{z}=-1.4 \mathrm{nT}$ ), the ionospheric projection of the cusp, $O_{S}^{\prime}$, was located in the pre-noon part of the southern polar cap boundary (due to the positive $B_{x}$ and negative $B_{y}$; $\varphi_{m} \approx 284^{\circ}$ ). Two unequal vortices converging on the singu- larity at $O_{S}^{\prime}$ are formed in the open field line region of the southern ionosphere (the dusk cell is dominant due to $B_{y}<0$ ). Figure 7B shows the convection patterns driven by the solar wind electric field at the beginning of the 25-31 January event and the corresponding Region 1 currents. Most of the auroral oval was connected with downward Region 1 currents, while the lesser, dawn part, near the singularity, was occupied by strong upward Region 1 currents. Calculations using Eq. (4) give the longitude range of the upward Region 1 currents as $256^{\circ} \leq \varphi \leq 284^{\circ}$.

Jumps in the solar wind magnetic field, density and velocity at the beginning of the 26-31 January event were much larger than those for the 16-17 January case. This is why the corresponding field-aligned currents were stronger for the major compression region starting on 26 January 2004.

Due to the southward IMF turning at the beginning of both of these events, the polar cap potential drop was applied to the ionospheric projection of the cusp (at $O_{S}^{\prime}$ in the southern Saturnian polar cap), where strong $S B Z$ currents were located. The polar cap arcs should be connected to these strong currents, as in the terrestrial magnetosphere for northward IMF. From observations at the Earth it has been found that the polar cap Sun-aligned arcs are located on the dawn side of the northern polar cap for $B_{y}<0$ and $B_{z}>0$ (e.g. Cumnock et al., 2002). By analogy, the same situation should occur in the southern kronian polar cap for $B_{y}<0$ and $B_{z}<0$, which is in good accordance with these observations (see Fig. 2k).

The characteristic time of the kronian transition current system can be estimated as the time necessary for the CIR front to pass through the open field line region at the magnetopause in the $x$-direction, $L_{x}$. During this time period (starting from the moment when the CIR front reaches the cusp regions), the polar cap area should decrease. For the first event we see from Fig. 1 that the solar wind pressure jump and simultaneous southward IMF rotation reach the dayside kronian magnetopause on 15 January 2004 at 12:00 UT. At that time, solar wind rarefaction conditions prevailed, so the subsolar distance, which we consider as a character magnetospheric scale, may be estimated to be of the order of $30 R_{S}$ (Alexeev et al., 2006). For the velocity $\sim 500 \mathrm{~km} \mathrm{~s}^{-1}$ observed in this case, the characteristic time for the terrestrial transition current system is $\sim 43 \mathrm{~min}$. Taking into account that the characteristic magnetospheric scale for Saturn for solar wind rarefaction conditions is $30 R_{S}$ and the corresponding value for the Earth is $10 R_{E}$, and that the planet's radius for Saturn is $\sim 10$ times larger than that of the Earth, we obtain that the characteristic passage time for Saturn to be $30 \times 43 \mathrm{~min}=22 \mathrm{~h}$. Thus, we assume that $22 \mathrm{~h}$ later than when the first solar wind pressure jump and simultaneous southward IMF rotation reached the dayside kronian magnetopause, the polar cap area began to contract. The growth of both the solar wind dynamic pressure and the negative $B_{N}$ continued during the next $20 \mathrm{~h}$ (see Fig. 1). So, we may expect that during the $\sim 42 \mathrm{~h}$ after the first shock encounter, the polar cap area was contracted. The first HST images on 
16 January 2004 were obtained $\sim 41 \mathrm{~h}$ after the shock is estimated to have hit the planet, and shows the auroral response at that time (Fig. 2f). Thus, we obtain good accordance with observations.

For the 26 January 2004 case, we see from Fig. 1 that the solar wind dynamic pressure was significantly higher in the second rarefaction region than in the first (prior to 16 January 2004). During the following few days, the magnetosphere was then further compressed by the CIR compression region. From the Pioneer 11 inbound flyby, we know that, for similar conditions, the compressed size of the dayside magnetosphere was of the order of $17 R_{S}$ (Smith et al., 1980). For similar high velocities, as those observed in this case $\left(\sim 540-620 \mathrm{~km} \mathrm{~s}^{-1}\right)$, the terrestrial transition current system's characteristic time is $31 \mathrm{~min}$ (Clauer et al., 2001). For Saturn, this time should be multiplied by $17 R_{S} / 10 R_{E} \sim 17$ (the ratio of the subsolar distance for Saturn to that of the Earth); thus, we obtain $17 \times 31 \mathrm{~min}=527 \mathrm{~min}=8.8 \mathrm{~h}$, which is in good accordance with observations: the time delay of the image obtained after the strong shock encounter (Fig. 2k) was $9 \mathrm{~h}$.

Thus, we have considered an additional mechanism for strong field-aligned current generation connected with the arrival of a compression region associated with a southward IMF rotation. We described why, in response to a sudden increase in the solar wind dynamic pressure with a simultaneous southward IMF turning, Saturn's aurora should move to higher latitudes, become brighter, and in the case of strong disturbances with $B_{y}<0$, the polar cap Sun-aligned arcs should fill the dawn side of the open field line region in the southern ionosphere.

\section{Discussion}

4.1 Field-aligned currents at the kronian open-closed field lines boundary

Discussing the field-aligned currents in the transition current system, we should mention that other field-aligned currents associated with the differential rotation between open and closed field lines also exist in the high-latitude kronian magnetosphere (Cowley et al., 2004a, b, 2005b). In Fig. 8 these two field-aligned current systems are shown. In the transition current system, the $S B Z$ currents, driven by the solar wind MHD-generator, are closed by the Region 1 fieldaligned currents. In the other current system, upward fieldaligned currents with linear density $I_{r}$, connected with the change in the ionospheric angular velocity at the boundary between open and closed field lines, flow along the boundary field lines to the magnetopause, and then close by return flow (not shown in Fig. 8) through the tail lobe downward to the open field line ionosphere (see Cowley et al., 2005b).

Let us estimate the $I_{r}$ currents due to shear in the ionospheric plasma angular velocity at the boundary between

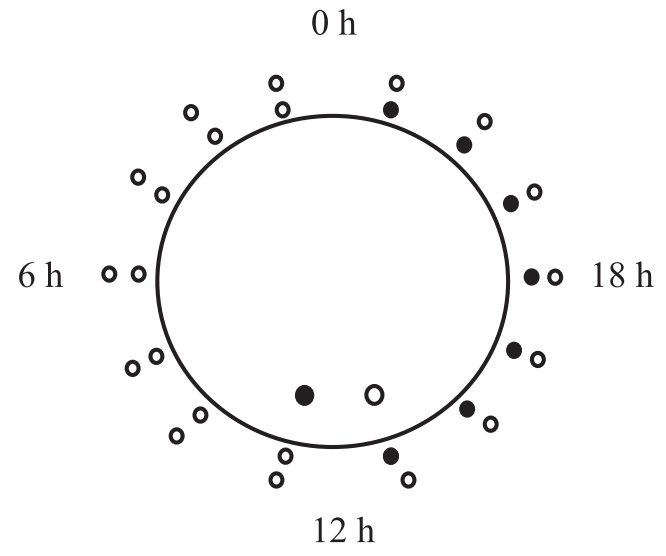

Fig. 8. Sketch of the kronian ionospheric projection of field-aligned currents for southward IMF. The field-aligned currents from pole to equator are: $S B Z$ (on open field lines), Region 1, and the upward field-aligned $I_{r}$-currents, connected with the change in the ionosheric angular velocity (both at the boundary of the open field line region). The boundary between open and closed field lines is marked by the large circle. The transition current system includes the $S B Z$ currents, ionospheric Pedersen currents in the open field line region (not shown), and the Region 1 field-aligned currents. Open circles indicate upward currents, while filled circles indicate downward currents.

open and closed field lines. The corotation electric field $\mathbf{E}_{\text {cor }}$ in the inertial magnetospheric spherical coordinate system is

$$
\mathbf{E}_{\text {cor }}=-\mathbf{V} \times \mathbf{B}=-\left[\gamma \boldsymbol{\Omega}_{S} \times \mathbf{r}\right] \times \mathbf{B}=-r \gamma \Omega_{S} \sin \theta \mathbf{e}_{\varphi} \times \mathbf{B},
$$

where $\mathbf{B}$ is the magnetic field, $\mathbf{r}$ is the radius-vector from Saturn's center, $\gamma$ is the fraction of "rigid rotation", $\Omega_{S} \approx 1.638 \cdot 10^{-4} \mathrm{~s}^{-1}$ is the angular velocity of Saturn, $\theta$ is the polar angle from Saturn's spin axis to $\mathbf{r}$, and $\mathbf{e}_{\varphi}$ is a unit vector in the azimuthal direction.

If for simplicity we assume that Saturn's surface magnetic field is dipole-like, such that $\mathbf{B}=\mathbf{B}_{\mathrm{d}}$, and $B_{\mathrm{d} \mathrm{r}}=2 B_{0 S}\left(\frac{R_{S}}{r}\right)^{3} \cos \theta, B_{\mathrm{d} \theta}=B_{0 S}\left(\frac{R_{S}}{r}\right)^{3} \sin \theta, B_{\mathrm{d} \varphi}=0$ $\left(B_{0 S}=21160 \mathrm{nT}\right.$ is the dipole field strength at Saturn's equator), we obtain:

$$
\begin{aligned}
& E_{\text {cor } r}=B_{0 S} \frac{R_{S}{ }^{3}}{r^{2}} \gamma \Omega_{S} \sin ^{2} \theta, E_{\text {cor } \theta}=-B_{0 S} \frac{R_{\mathrm{S}}{ }^{3}}{r^{2}} \gamma \Omega_{\mathrm{S}} \sin 2 \theta, \\
& E_{\text {cor } \varphi}=0 .
\end{aligned}
$$

At the polar cap boundary $\left(\theta=\theta_{m}\right)$ only one component of the corotation electric field $E_{\mathrm{cor} \theta}$ changes, creating a corresponding change in the ionospheric Pedersen current, $\mathbf{I}_{P}$ :

$\left.\Sigma_{P}^{*}\left\{E_{\operatorname{cor} \theta}\right\}\right|_{\theta_{m}}=\left.\left\{\mathbf{I}_{P}\right\}\right|_{\theta_{m}}$.

Latitudinal variations in $\mathbf{I}_{P}$ are associated with the fieldaligned current density, $j_{n}$, whose jump, $\left\{j_{n}\right\}$ is given by

$\left.\left\{j_{n}\right\}\right|_{\theta_{m}}=-\left.\operatorname{div} \mathbf{I}_{P}\right|_{\theta_{m}}$, 
where $\operatorname{div} \mathbf{I}_{P}$ is a two-dimensional divergence. Assuming that under the ionosphere, field-aligned currents are absent, we obtain $\left.\left\{j_{n}\right\}\right|_{\theta_{m}}=\left.j_{n}\right|_{\theta_{m}}$, where $\left.j_{n}\right|_{\theta_{m}}$ is the field-aligned current density above the ionosphere at the open-closed field line boundary. As $E_{\text {cor } r}$ and $E_{\operatorname{cor} \varphi}$ are continuous at $\theta=\theta_{m}$, we obtain:

$$
\left.\operatorname{div} \mathbf{I}_{P}\right|_{\theta_{m}}=\left.\Sigma_{P}^{*} \operatorname{div} \mathbf{E}_{\text {cor }}\right|_{\theta_{m}}=\left.\frac{\Sigma_{P}^{*}}{R_{S} \sin \theta} \frac{\partial}{\partial \theta}\left(E_{\operatorname{cor} \theta} \sin \theta\right)\right|_{\theta_{m}} .
$$

The Pioneer and Voyager observations presented by Frank et al. (1980) and Richardson (1986) indicate that the plasma on outer closed field lines rotates with an angular velocity which is $\sim 50 \%-80 \%$ of the rigid rotation, while according to the results of Stallard et al. (2004), obtained from infrared Doppler observations, the rotational flow on open field lines is $\sim 20 \%-30 \%$ of the rigid corotation. Thus, taking into account that parameter $\gamma$ is different on the open $\left(\gamma_{o} \sim 0.25\right)$ and closed $\left(\gamma_{c} \sim 0.65\right)$ field lines, we obtain at the polar cap boundary:

$$
\begin{aligned}
& \left.\frac{1}{\sin \theta} \frac{\partial}{\partial \theta}\left(E_{\operatorname{cor} \theta} \sin \theta\right)\right|_{\theta_{m}}=\left.\frac{1}{\delta \theta \sin \theta}\left\{E_{\operatorname{cor} \theta} \sin \theta\right\}\right|_{\theta_{m}} \\
& =-B_{0 S} \delta \gamma \Omega_{S} R_{S} \sin 2 \theta_{m}(\delta \theta)^{-1},
\end{aligned}
$$

where $\delta \gamma=\gamma_{c}-\gamma_{o} \sim(0.65-0.25) \sim 0.4$, and $\delta \theta$ is the width of the ring of the upward field-aligned current. From Eqs. (8), (9) and (10) the expression for $j_{n}$ and $\operatorname{div} \mathbf{I}_{P}$ at $\theta=\theta_{m}$ can be obtained:

$$
\left.j_{n}\right|_{\theta_{m}}=-\left.\operatorname{div} \mathbf{I}_{P}\right|_{\theta_{m}}=\Sigma_{P}^{*} B_{0 S} \delta \gamma \Omega_{\mathrm{S}} \sin 2 \theta_{m}(\delta \theta)^{-1} .
$$

It is seen that an upward (positive) field-aligned current with density $\left.j_{n}\right|_{\theta_{m}}>0$ arises along the whole boundary of the polar cap. Integrating this current density $\left.j_{n}\right|_{\theta_{m}}$ over the band of the upward field-aligned currents with an area $S_{f}$, we find the total field-aligned current strength $J_{r}$. In a zero approximation $S_{f}=2 \pi R_{S}^{2} \delta \theta \sin \theta_{m}$, and

$$
\begin{aligned}
& J_{r}=\left.j_{n}\right|_{\theta_{m}} \cdot S_{f}=2 \pi \Sigma_{P}^{*} B_{0 \mathrm{~S}} \delta \gamma \Omega_{\mathrm{S}} R_{S}^{2} \sin 2 \theta_{m} \sin \theta_{m}= \\
& 2 \pi \Sigma_{P}^{*} B_{i} \delta \gamma \Omega_{\mathrm{S}} R_{S}^{2} \sin ^{2} \theta_{m} \cos \theta_{m},
\end{aligned}
$$

where $B_{i}=2 B_{0 \mathrm{~S}}$ is the strength of the polar ionospheric magnetic field. We take $\theta_{m}$ values of $\sim 13^{\circ}$ and $\sim 10^{\circ}$ for the 16 and 26 January 2004 events, respectively. These overall values were obtained from the open flux estimates determined by Badman et al. (2005) (of 24 and $13 \mathrm{GWb}$, respectively). By substituting these values and $\Sigma_{P}^{*}=1$ mho into Eq. (12), one obtains total boundary currents associated with the differential rotation of open and closed field lines of $\sim 3 \mathrm{MA}$ and $\sim 2$ MA for 16 and 26 January, respectively. These values are in accord with the estimates made previously by Cowley et al. (2004a, b).

The MHD solar wind generator creating the $S B Z$ currents, located at the cusp ionospheric projection, can be charaterized by a polar cap potential drop $\delta \Phi_{p c}$ (also located at the cusp projection) which is determined in terms of the solar wind parameters as follows

$\delta \Phi_{p c}=k_{S} B_{t r} V_{s w} L_{t r}$,

where $B_{t r}=\left(B_{z}{ }^{2}+B_{y}{ }^{2}\right)^{1 / 2}$ (e.g. Clauer et al., 2001), $k_{S}$ is a coefficient of the IMF penetration into Saturn's magnetosphere, $B_{z}$ and $B_{y}$ are the north-south and azimuthal IMF components, respectively, and $L_{t r}$ is the width of the open field line "window" at the magnetopause. In both of the cases discussed, the IMF $B_{y}$ component at the time of the images "f" and "k" in Figs. 1 and 2, was large. It created a "window" width of the order of $3 R_{S S} \sim 60 R_{S}$, where the average distance of the subsolar point, $R_{S S}$, is $20 R_{S}$ (see Clauer et al. (2001) for the analogous case in the terrestrial magnetosphere). $V_{s w}$ was $\sim 530 \mathrm{~km} / \mathrm{s}$ for 16 January and $\sim 620 \mathrm{~km} / \mathrm{s}$ for 26 January 2004; $B_{z}$ and $B_{y}$ were $-0.4 \mathrm{nT}$ for $16 \mathrm{Jan}-$ uary, and $B_{z}=-1.4 \mathrm{nT}$ and $B_{y}=-2 \mathrm{nT}$ for 26 January (see Fig. 1). The value of $k_{S}\left(0<k_{S}<1\right)$ is not known with any certainty.

Modelling the Jovian magnetosphere dependence on the solar wind magnetic field, Belenkaya (2004) showed that for a typical value of $B_{z}=-0.5 \mathrm{nT}, k_{S} \sim 0.8$ gives good accordance with observations. The scale of the anti-corotation region in the noon outer low-latitude Jovian magnetosphere can be estimated as $8 R_{\mathrm{J}}$ from the data published by Desai and Simnett (1996) and as $6 R_{\mathrm{J}}$ from the data given by Cowley et al. (1996). Both of these estimates lead to the conclusion that the value of $k_{J}=0.8$ (corresponding to the model scale $5.3 R_{J}$, where $R_{J}$ is the Jupiter's radius) is most appropriate for the interpretation of observations of the solar wind-driven plasma flows. The chosen southward direction of the IMF was close to the one (with the strength $\sim 0.5 \mathrm{nT}$ ) which was directly measured by Ulysses in February 1992 (e.g. Staines at al., 1993; Cowley et al., 1996). Here we also note that Tsyganenko (2002) found the best correspondence between his model of the near-Earth magnetosphere and observational data for the coefficient of IMF penetration into the Earth's magnetosphere of between 0.15 and 0.8 .

If we follow Belenkaya (2004) and choose $k_{S} \sim 0.8$ (as for Jupiter), then we obtain $\delta \Phi_{p c} \sim 0.9 \mathrm{MV}$ for 16 January, and $\sim 14.8 \mathrm{MV}$ for 26 January 2004 . If we assume instead that $k_{S}$ is closer to the Earth's case, for example, $k_{S} \sim 0.2$, then $\delta \Phi_{p c} \sim 0.2 \mathrm{MV}$ for 16 January, and $\sim 3.7 \mathrm{MV}$ for 26 January 2004. (An analytical finite-conductivity solution for the IMF penetration into the terrestrial magnetosphere was obtained by Alexeev, 1986).

These values of the polar cap potential drop $\delta \Phi_{p c}$ (0.9-0.2 MV for 16 January and 14.8-3.7 MV for 26 January 2004), caused by the MHD-generator of the solar wind, should be compared with the potential drop between the pole and the polar cap boundary $\left(\theta_{m}\right)$ generated by the planet's rotation, $\delta U_{p c-\text { cor }}$. Using the method described by Belenkaya (2004) for Jupiter, we can find the expression for the 


\section{Geometric factor}

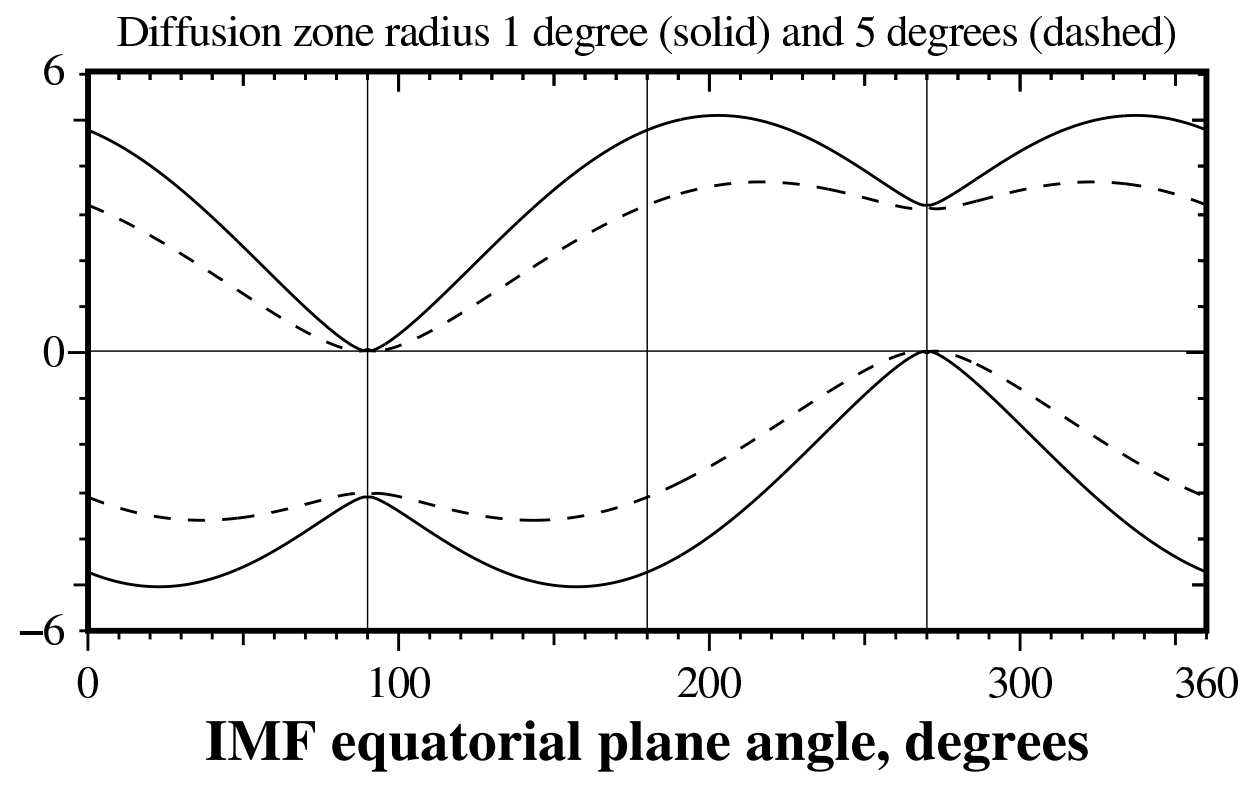

Fig. 9. Geometric factor, $\epsilon$, of the distribution of field-aligned current, $J_{0}$, along the open-closed field line boundary in the transition current system. For two diffusion zone (region of the cusp field-aligned currents location) radii $\delta=1^{\circ}$ (solid) and $\delta=5^{\circ}$ (dashed), the upper curves show the dependence for the upward current, and the bottom curves show the dependence for the downward current on the IMF equatorial plane angle $\varphi_{m}$ (Belenkaya et al., 2004).

ionospheric corotation potential in the open field line region as follows

$U_{p c-\text { cor }}=\gamma_{o} \Omega_{S} B_{0 S} R_{S}^{2} \sin ^{2} \theta+C$,

where $C$ is a constant. Then,

$\delta U_{p c-\text { cor }}=\gamma_{o} \Omega_{S} B_{0 S} R_{S}^{2} \sin ^{2} \theta_{m}$.

Taking into account that $\gamma_{o}=0.25$ and $\sin \theta_{m}$ is equal to 0.22 for 16 January and 0.17 for 26 January 2004, we obtain $\delta U_{p c-\text { cor }} \sim 0.5 \mathrm{MV}$ for these two events. From these estimations we see that $\delta \Phi_{p c}$ was of the order of, or higher than, $\delta U_{p c-\text { cor }}$ for the cases considered. So, corotation effects should be taken into account during the investigation of the IMF influence on the Saturnian high-latitude magnetosphere/ionosphere.

The influence of the IMF's direction on Saturn's aurora considered here is associated with the total strength of the field-aligned current $J_{0}$ in the transition current system. The total integrated field-aligned current $J_{0}$ can be written as

$J_{0}=\int I_{\|} \cdot R_{S} \sin \theta_{m} d \varphi$.

Integration is made along the arc of the circle $\theta=\theta_{m}$ in which the sign of $I_{\|}$is constant. Taking the linear current density $I_{\|}$ from Eq. (2), we obtain

$J_{0 \pm}=-\Sigma_{P}^{*} \delta \Phi_{p c} \epsilon_{ \pm}, \quad \epsilon=\frac{1}{2} \int\left[\sin \varphi-\frac{\cos \varphi \cot \frac{\varphi_{m}-\varphi}{2}}{\cos \theta_{m}}\right] d \varphi$.
Here $\epsilon_{ \pm}$is a geometric factor, determining the distribution of the total upward and downward $\left(J_{0 \pm}\right)$ current at the ionospheric level (see Fig. 9). From Fig. 9 it follows that for the values of $\varphi_{m}$ in these two discussed events $\left(\varphi_{m}=3 \pi / 2\right.$ for 16 January and $\varphi_{m} \approx 284^{\circ}$ for 26 January 2004), the geometric factor $\epsilon$ was of the order of 3 . The parameter $\epsilon$ determines the scheme of connection of the MHD solar wind generator with the ionosphere for an IMF antiparallel to the planet's dipole. Thus, it is the same for the Earth and Saturn under similar conditions.

From Eq. (17) for $\Sigma_{P}^{*} \sim 1$ mho, $\epsilon \sim 3$, and the determined values of $\delta \Phi_{p c}(0.9-0.2 \mathrm{MV}$ for 16 January and 14.8-3.7 MV for 26 January 2004), we obtain $J_{0}$ of the order of 2.7-0.6 MA for 16 January and 44.4-11.1 MA for 26 January 2004. If we compare the field-aligned current strengths in the transition current system, $J_{0}$, and in the current system caused by the differential rotation, $J_{r}(\sim 3 \mathrm{MA}$ and $\sim 2 \mathrm{MA}$ for the 16 and 26 January 2004, respectively), we have to conclude that the currents are comparable, so that generally their combined effect needs to be considered. In the Jackman and Cowley (2006) model the Region 1 fieldaligned currents also produce a significant modulation of the field-aligned currents associated with differential rotation.

Thus, the near-uniform upward field-aligned boundary currents caused by differential rotation on open and closed field lines must be added to those associated with the IMFdependent solar wind-driven convection. The overall current 
system for 16 January will take the form of strong upward currents of the order of 5.7-3.6 MA near local dawn (connected with bright aurora) and more weak upward currents 0.3-2.4 MA at dusk (associated with weaker aurora). The whole polar cap area should decrease. The " $\mathrm{f}$ " image in Fig. 2 corresponds to this picture.

For 26 January 2005 the total field-aligned current structure will include very strong upward field-aligned currents of the order of 46.4-13.1 MA in the dawn-prenoon sector, and downward currents 42.4-9.1 MA in the dusk sector. The polar cap should be significantly contracted. So, such huge upward currents at dawn (probably exceeding the threshold value) should be associated with strong field-aligned potential drops and corresponding very bright auroras (a detailed investigation of the relation between field-aligned currents of arbitrary large amplitude and corresponding field-aligned voltages has recently been presented by Cowley, 2006, following earlier work by Knight, 1973). The SBZ currents connected with these large Region 1 currents create bright polar arcs. On the dusk side the presence of strong downward currents leads to the absence of aurora. Image " $\mathrm{k}$ " in Fig. 2 supports this conclusion. We see bright Sun-aligned polar arcs and a bright oval on the dawn side and an almost invisible duskside portion of the contracted auroral oval.

\subsection{Responses of auroral activity to interplanetary shocks at Earth and Saturn}

For the Earth it was found that when southward IMF occurs prior to the impact of an interplanetary shock, substorms arise with a high probability, and a current disruption and/or tail magnetic field reconnection can be triggered (Zhou and Tsurutani, 2001; Tsurutani and Zhou, 2003). Northward IMF prior to interplanetary shocks does not lead to midnight auroral activity, but increases the magnetic latitude of the auroral oval (e.g. Zhou and Tsurutani, 2001). We see that at Saturn, for the cases studied, the IMF at the shock was antiparallel to the planet's magnetic dipole moment, and corresponding effects were observed. The auroral oval occurred on higher magnetic latitudes, and there was no increase in the midnight auroral activity associated with interplanetary shocks. Most likely, the direction of the southward-northward IMF component prior to the interplanetary shock compression of the magnetosphere is significant, not only for the terrestrial magnetospheric activity (see Zhou and Tsurutani, 2001; Tsurutani and Zhou, 2003) but also for the kronian activity. However, at the present time, we do not have enough data to support or refute this suggestion. Prangé et al. (2004) used the HST images found on 7 December 2000 of an unusual aurora which they identified with the first manifestation of an auroral storm at Saturn. They associated this phenomenon with the passage of an interplanetary shock, or more correctly, with a series of CME-driven shocks, detected near the Earth in November 2000 (at that time, the Sun, the Earth, Jupiter and Saturn were nearly aligned). However, corresponding solar wind measurements near Saturn, which could characterize the passage of shocks directly, were absent.

Zhou and Tsurutani (1999) and Tsurutani et al. (2001) noted that significant charged-particle precipitation occurs in the dayside auroral zone during and after interplanetary shock impingements on the Earth's magnetosphere. They proposed the following mechanisms for particle energization and precipitation immediately after shock compression of the magnetosphere. (1) Betatron compression of pre-existing outer zone magnetospheric particles. The anisotropic plasma is unstable to loss-cone instabilities, leading to plasma wave growth, resonant particle pitch-angle scattering and electron and proton losses into the upper ionosphere. (2) The compression of the magnetosphere can also lead to enhanced field-aligned currents and the formation of dayside doublelayers. (3) In the latter stages of the storm's initial phase, there is evidence of a long-lasting, viscous-like interaction occurring on the flanks of the magnetopause. It was noted that the dayside auroral brightenings occur just after the shock compression of the frontside magnetosphere.

If hot trapped plasma exists in Saturn's outer magnetosphere, then a similar phenomenon as observed at Earth could happen in the kronian ionosphere, that is, auroral brightening at noon followed by propagation of the shock front around both dawn and dusk to the nightside. At the present time, we have no observations which support this, but such a situation seems, in principle, possible, with some modifications due to Saturn's specific characteristics. The HST images in January 2004 constitute only a relatively sparse series of snapshots, so even if short-term noon brightenings did occur after the two shock encounters, they could occur in intervals when no HST observations are available.

Prangé et al. (2004) described the observed "storm" auroral activity in the kronian ionosphere on 7 December 2000. During the storm, Saturn's auroral oval remained almost identical in size and location, but it was globally brighter by $50 \%$. Its midnight-to-dawn sector became very active (almost four times its usual brightness). In addition, there was a very bright feature inside the dawnside oval. It extended polewards up to $\sim 78^{\circ} \mathrm{S}\left(\sim 6^{\circ}\right.$ poleward of the oval), and is confined to the 05:30-11:00 local time sector. Its peak brightness largely exceeds that of the oval, and its total auroral output is comparable to the oval output, despite its very limited spatial extent. Prangé et al. (2004) noted that this kronian polar cap bright feature is reminiscent of the terrestrial noon polar cusp, frequently observed during dayside reconnection; however, at Saturn the analog of the "cusp brightening" occurred in the morning sector. Prangé et al. (2004) also emphasized that Saturn's oval does not exhibit the expansion towards lower latitudes which is typical of the geomagnetic storm oval. 


\section{Summary}

Cassini magnetic field and plasma data obtained in the interplanetary medium in January 2004 (Crary et al., 2005; Bunce et al., 2006) are used to investigate the solar wind interaction with the kronian magnetosphere. The HST images of Saturn's aurora, obtained simultaneously in the southern polar cap corresponding to the arrival of the 16-17 January and 2631 January 2004 compression regions (Clarke et al., 2005; Bunce et al., 2006), are considered as a possible manifestation of two mechanisms acting in Saturn's magnetosphere.

The first mechanism is connected with the onset of tail reconnection triggered by the sudden compression of the magnetosphere (Cowley et al., 2005a; Bunce et al., 2006). Here we propose that the polarisation current $i_{p}$ directed to dusk at the wave front of the compressed region in the dayside kronian magnetosphere may be closed by the equatorial current on the disturbed magnetopause (enhanced Chapman-Ferraro current). The resulting equatorial current system, which we call a "transient ring current", creates a magnetic field in the noon-midnight meridional cross section, which is directed southward inside the compressed region and northward outside of it. In the kronian tail current sheet, near its inner edge, the southward background magnetospheric magnetic field is weakened by the northward magnetic field of the tail current system. This produces a preferable place where the appropriate conditions for tail reconnection could occur. Thus, the onset of tail reconnection, triggered by the sudden compression of the magnetosphere (Cowley et al., 2005a; Bunce et al., 2006), arises. This will cause the open-closed field line boundary to contract poleward, initially on the nightside, producing bright auroras in the closed field "bulge" in a manner similar to terrestrial substorms. The auroras will be due both to the production and precipitation of hot plasma from the tail, and to the excitation of associated field-aligned current systems. As time goes on, the hot plasma and associated precipitation will sub-corotate around the outer magnetosphere via dawn, due to magnetosphere-ionosphere coupling to the rotating upper atmosphere.

In addition to these compression-induced tail effects, the distribution of currents and related auroras will also be simultaneously affected by the on-going, IMF-dependent interaction of the magnetosphere with the solar wind at the magnetopause. For the conditions which occurred during the January 2004 observations, we suggest that a transition current system was excited, which is analogous to that described for the terrestrial system by Clauer et al. (2001) and Belenkaya et al. (2004). In the Earth's magnetospere, the transition current system arose after the arrival of a strong solar wind pressure pulse accompanied by a simultaneous northward turning of the IMF, corresponding to a southward turn at Saturn, as occurred during the January 2004 events. The three-dimensional kronian current system includes the Region 1 field-aligned currents concentrated at the open-closed field line boundary, the $S B Z$ field-aligned currents distributed over the polar cap near the cusp (the Saturnian $S B Z$ field-aligned currents existing for the southward IMF are similar to the $N B Z$ currents for the Earth, arising under the northward IMF conditions), and the ionospheric Pedersen currents on open field lines, closing both of these field-aligned currents. This current system will be added to the ring of upward currents of comparable magnitude at the boundary which is associated with the differential rotation of the plasma between open and closed field lines, such that the overall current system for the events studied in January 2004 generally takes the form of strong upward field-aligned currents on the dawn side, with corresponding bright aurora, and weaker upward, or even downward field-aligned currents at dusk with weaker, or perhaps without any auroras. The polar cap area is reduced. It is suggested that the combination of the tail reconnection effects discussed above, and the IMFmodulated solar wind interaction both play a role in the auroral dynamics during the January 2004 compression events.

Acknowledgements. Work at INP Moscow State University was supported by INTAS Grant No 03-51-3922 and by the RFBR Grants 04-05-64396 and 05-05-64435. Work at Leicester was supported by PPARC Grant PPA/G/0/2003/00013. We thank S. Badman for providing Fig. 1, containing data from the MAG and CAPS investigations on the Cassini spacecraft. The magnetic field data were obtained courtesy of M. Dougherty and the MAG team at Imperial College, while the plasma data were obtained through F. Crary and the CAPS team. Figure 2 employs observations made by the NASA/ESA Hubble Space Telescope, obtained at the Space Telescope Science Institute, which is operated by the AURA Inc for NASA. The images were kindly provided by J. Clarke.

Topical Editor I. A. Daglis thanks I. Dandouras and another referee for their help in evaluating this paper.

\section{References}

Alexeev, I. I.: The penetration of interplanetary magnetic and electric fields into the magnetosphere, J. Geomag. Geoelectr., 38, 1199-1221, 1986.

Alexeev, I. I. and Belenkaya, E. S.: Electric field in an open model of the magnetosphere, Geomagn. Aeron., 23, 57-61, 1983.

Alexeev, I. I. and Belenkaya, E. S.: Convection of the magnetospheric plasma on the open field line region, Geomagn. Aeron. (in Russian), 25(3), 450-457, 1985.

Alexeev, I. I., Kalegaev, V. V., Belenkaya, E. S., Bobrovnikov, S. Y., Bunce, E. J., Cowley, S. W. H., and Nichols, J. D.: A global magnetic model of Saturn's magnetosphere, and a comparison with Cassini SOI fly-through data, Geophys. Res. Lett., 33, L08101, doi:10.1029/2006GL025896, 2006.

Badman, S. V., Bunce, E. J., Clarke, J. T., Cowley, S. W. H., Gérard, J.-C., Groden, D., and Milan, S. E.: Open flux in Saturn's magnetosphere during the January 2004 Cassini-HST campaign, and implications for reconnection rates, J. Geophysical Res., 110, A11216, doi:10.1029/2005JA011240, 2005.

Behannon, K. W., Lepping, R. P., and Ness, N. F.: Structure and dynamics of Saturn's outer magnetosphere and boundary regions, J. Geophys. Res., 88, 8791-8800, 1983. 
Belenkaya, E. S.: Comment on "Observations of reconnection of interplanetary and lobe magnetic field lines at the high-latitude magnetopause" by Gosling, J. T., Thomsen, M. F., Bame, S. J., Elphic, R. C., and Russell, C. T., J. Geophys. Res., 98, 59415944, 1993.

Belenkaya, E. S.: Reconnection modes for near-radial IMF, J. Geophys. Res., 103, 26487-26494, 1998a.

Belenkaya, E. S.: High-latitude ionospheric convection patterns dependent on the variable IMF orientation, J. Atmos. Solar-Terr. Phys., 60, 1343-1354, 1998b.

Belenkaya, E. S.: The jovian magnetospheric magnetic and electric fields: effects of the interplanetary magnetic field, Planet. Space Sci., 52, 499-511, 2004.

Belenkaya, E. S., Alexeev, I. I., and Clauer, C. R.: Field-aligned current distribution in the transition current system, J. Geophys. Res., 109, A11207, doi:10.1029/2004JA010484, 2004.

Blanc, M., Bolton, S., Bradley, J., Burton, M., Cravens, T. E., Dandouras, T., Dougherty, M. K., Festou, M. C., Feynman, J., Johnson, R. E., Gombosi, T. G., Kurth, W. S., Liewers, P. C., Mauk, B. H., Maurice, S., Mitchells, D., Neubauer, F. M., Richardson, J. D., Shemansky, D. E., Sittler, E. C., Tsurutani, B. T., Zarka, Ph., Esposito, L. W., Grun, E., Gurnett, D. A., Kliore, J. A., Krimigis, S. M., Southwood, D., Waite, J. H., and Young, D. T.: Magnetospheric and plasma science with Cassini-Huygens, Space Sci. Rev., 104, 253-346, 2002.

Bunce, E. J., Cowley, S. W. H., and Wild, J. A.: Azimuthal magnetic fields in Saturn's magnetosphere: Effects associated with plasma sub-corotation and magnetopause-tail current system, Ann. Geophys., 21, 1709-1722, 2003.

Bunce, E. J., Cowley, S. W. H., Jackman, C. M., Clarke, J. T., Crary, F. J., and Dougherty, M. K.: Cassini observations of the interplanetary medium upstream of Saturn and their relation to Hubble Space Telescope aurora data, Adv. Space Res., in press, 2006.

Clarke, J. T., Gérard, J.-G., Grodent, D., Wannawichian, S., Gustin, J., Connerney, J., Crary, F., Dougherty, M., Kurth, W., Cowley, S. W. H., Bunce, E. J., Hill, T., and Kim, J.: Morphological differences between Saturn's ultraviolet aurorae and those of Earth and Jupiter, Nature, 433, 717-719, 2005.

Clauer Jr., C. R., Alexeev, I. I., Belenkaya, E. S., and Baker, J. B.: Special features of the September 24-27, 1998 storm during high solar wind dynamic pressure and northward interplanetary magnetic field, J. Geophys. Res., 106, 25 695-25 712, 2001.

Cowley, S. W. H.: A qualitative study of the reconnection between the Earth's magnetic field and an interplanetary field of arbitrary orientation, Radio Sci., 8, 903-913, 1973.

Cowley, S. W. H.: Current-voltage and kinetic energy flux relations for relativistic field-aligned acceleration of auroral electrons, Ann. Geophys., 24, 325-338, 2006.

Cowley, S. W. H. and Bunce, E. J.: Corotation driven magnetosphere-ionosphere coupling currents in Saturn's magnetosphere and their relation to the auroras, Ann. Geophys., 21, 1691-1707, 2003.

Cowley, S. W. H., Balogh, A., Dougherty, M. K., Dunlop, M. W., Edwards, T. M., Forsyth, R. J., Hynds, R. J., Laxton, N. F., and Staines, K.: Plasma flow in the Jovian magnetosphere and related magnetic effects: Ulysses observations, J. Geophys. Res., 101, 15 197-15 210, 1996.

Cowley, S. W. H., Bunce, E. J., and Prangé, R.: Saturn's polar ionospheric flows and their relation to the main auroral oval, Ann.
Geophys., 22, 1379-1394, 2004a.

Cowley, S. W. H., Bunce, E. J., and O'Rourke, J. M.: A simple quantitative model of plasma flows and currents an Saturn's polar ionosphere, J. Geophys. Res., 109, A05212, doi:10.1029/2003JA010375, 2004b.

Cowley, S. W. H., Badman, S. V., Bunce, E. J., Clarke, J. T., Gérard, J.-C., Jackman, C. M., Milan, S. E., and Yeoman, T. K.: Reconnection in a rotation-dominated magnetosphere and its relation to Saturn's auroral dynamics, J. Geophys. Res., 110, A02201, doi:10.1029/2004JA010796, 2005a.

Cowley, S. W. H., Alexeev, I. I., Belenkaya, E. S., Bunce, E. J., Cottis, C. E., Kalegaev, V. V., Nichols, J. D., Prangé, R., and Wilson, F. J.: A simple axi-symmetric model of magnetosphereionosphere coupling currents in Jupiter's polar ionosphere, J. Geophys. Res., 110, A11209, doi:10.1029/2005JA011237, 2005b.

Crary, F. J., Clark, J. T., Dougherty, M. K., Hanlon, P. G., Hansen, K. C., Steinberg, J. T., Barrachlough, B. L., Coates, A. J., Gerard, J.-C., Groden, D., Kurth, W. S., Mitchell, D. G., Rymer, A. M., and Young, D. T.: Solar wind dynamic pressure and electric field as the main factors controlling Saturn's auroras, Nature, 433, 720-722, 2005.

Cumnock, J. A., Sharber, J. R., Heelis, R. A., Blomberg, L. G., Germany, G. A., Spann, J. F., and Coley, W. R.: Interplanetary magnetic field control of theta aurora development, J. Geophys. Res., 107, A7, doi:10.1029/2001JA009126, 2002.

Desai, M. I. and Simnett, G. M.: Solar wind-driven flows in the Jovian magnetosphere, J. Geophys. Res., 101, 13 115-13 135, 1996.

Dougherty, M. K., Kellock, S., Southwood, D. J., Balogh, A., Smith, E. J., Tsurutani, B. T., Gerlach, B., Glassmeier, K. H., Gleim, F., Russell, C. T., Erdos, G., Neubauer, F. M., and Cowley, S. W. H.: The Cassini magnetic field investigation, Space Sci. Rev., 114, 331-383, 2004.

Frank, L. A., Burek, B. G., Ackerson, K. L., Wolfe, J. H., and Mihalov, J. D., Plasmas in Saturn's magnetosphere, J. Geophys. Res., 85, 5695-5708, 1980.

Gérard, J.-C., Grodent, D., Gustin, J., and Saglam, A.: Characteristics of Saturn's FUV aurora observed with the Space Telescope Imaging Spectrograph, J. Geophys. Res., 109, A09207, doi:10.1029/2004JA010513, 2004.

Jackman, C. M. and Cowley, S. W. H.: A model of the plasma flow and current in Saturn's polar ionosphere under conditions of strong Dungey-cycle driving, Ann. Geophys., in press, 2006.

Jackman, C. M., Achilleos, N., Bunce, E. J., Cowley, S. W. H., Dougherty, M. K., Jones, G. H., and Milan, S. E.: Interplanetary magnetic field at $\sim 9 \mathrm{AU}$ during the declining phase of the solar cycle and its implications for Saturn's magnetospheric dynamics, J. Geophys. Res., 109, A11203, doi:10.1029/2004JA010614, 2004.

Knight, S.: Parallel electric fields, Planet. Space Sci., 21, 741-750, 1973.

Lepping, R. P., Sittler, E. C., Mish, W. H., Curtis, S. A., and Tsurutani, B. T.: Analysis of waves in Saturn's dayside magnetosphere: Voyager 1 observations, J. Geophys. Res., 110, A05201, doi:10.1029/2004JA010559, 2005.

Maclennan, C. G., Lanzerotti, L. J., Krimigis, S. M., and Lepping, R. P.: Low-energy particles at the bow shock, magnetopause, and outer magnetosphere of Saturn, J. Geophys. Res., 88, A11, 
8817-8830, 1983.

Maltsev, Yu. P. and Ostapenko, A. A.: Variability of the electric currents in the magnetosphere, Phys. Chem. Earth, (C), 25(1-2), 27-30, 2000.

Maurice, S. and Engel, I. M.: Idealized Saturn magnetosphere shape and field, J. Geophys. Res., 100, 17 143-17 151, 1995.

Milan, S. E.: A simple model of the flux content of the distant magnetotail, J. Geophys. Res., 109, A07210, doi:10.1029/2004JA010397, 2004.

Prangé, R., Pallier, L., Hansen, K. C., Howard, R., Vourlidas, A., Courtin, R., and Parkinson, C.: An interplanetary shock traced by planetary auroral storms from the Sun to Saturn, Nature, 432, 78-81, 2004.

Richardson, J. D.: Thermal ions at Saturn: Plasma parameters and implications, J. Geophys. Res., 91, 1381-1389, 1986.

Russell, C. T.: The configuration of the magnetosphere, in: Critical Problems of Magnetospheric Physics, edited by: Dyer, E. R., p. 1-16, Inter-Union Comm. on Sol. Terr. Phys., Ntl. Acad. of Sci., Washington, D.C., 1972.

Smith, E. J., Davis, L., Jones, D. E., Coleman, P. J., Colburn, D. S. Dyal, P., and Sonett, C. P.: Saturn's magnetosphere and its interaction with the solar wind, J. Geophys. Res., 85, A11, 56555674, 1980.

Staines, K., Balogh, A., Cowley, S. W. H., Edwards, T. M., Forsith, R. J., and Hynds, R. J.: Ulysses observations of noncorotational flows in the outer dayside Jovian magnetosphere, Planet. Space Sci., 41, 931-946, 1993.

Stallard, T. S., Miller, S., Trafton, L. M., Geballe, T. R., and Joseph, R. D.: Ion winds in Saturn's southern auroral/polar region, Ikarus, 167, 204-211, 2004.

Tamao, T.: Unsteady interactions of solar wind disturbances with the magnetosphere, J. Geophys. Res., 80, 4230-4236, 1975.

Tsurutani, B. T. and Zhou, X.-Y.: Interplanetary shock triggering of substorms: Wind and Polar, Adv. Space Res., 31(4), 1063-1067, 2003.
Tsurutani, B. T., Zhou, X.-Y., Arballo, J. K., Gonzalez, W. D., Lakhina, G. S., Vasyliunas, V., Pickett, J. S., Araki, T., Yang, H., Rostoker, G., Hughes, T. J., Lepping, R. P., and Berdichevsky, D.: Auroral zone dayside precipitation during magnetic storm initial phases, J. Atmos. S.-P., 63, 513-522, 2001.

Tsyganenko, N. A.: A model of the near magnetosphere with a dawn-dusk asymmetry. Mathematical structure, J. Geophys. Res., 107, A8, doi:10.1029/2001JA000219, 2002.

Wilken, B., Goertz, C. K., Baker, D. N., Higbie, P. R., and Fritz, T. A.: The SSC on July 29, 1977 and its Propagation Within the Magnetosphere, J. Geophys. Res., 87, A8, 5901-5910, 1982.

Young, D. T., Berthelier, J. J., Blanc, M., Burch, J. L., Coates, A. J., Goldstein, R., Grande, M., Hill, T. W., Johnson, R. E., Kelha, V., McComas, D. J., Sittler, E. C., Svenes, K. R., Szego, K., Tanskanen, P., Ahola, K., Anderson, D., Bakshi, S., Baragiola, R. A., Barraclough, B. L., Black, R. K., Bolton, S., Booker, T., Bowman, R., Casey, P., Crary, F. J., Delapp, D., Dirks, G., Eaker, N., Funsten, H., Furman, J. D., Gosling, J. T., Hannula, H., Holmlund, C., Huomo, H., Illiano, J. M., Jensen, P., Johnson, M. A., Linder, D. R., Luntama, T., Maurice, S., McCabe, K. P., Mursula, K., Narheim, B. T., Nordholt, J. E., Preece, A., Rudzki, J., Ruitberg, A., Smith, K., Szalai, S., Thomsen, M. F., Viherkanto, K., Vilppola, J., Vollmer, T., Wahl, T. E., Wuest, M., Ylikoppi, T., and Zinsmeyer, C.: Cassini plasma spectrometer investigation, Space Sci. Rev., 114, 1-112, 2004.

Zhou, X.-Y. and Tsurutani, B. T.: Rapid intensification and propagation of the dayside aurora: Large scale interplanetary pressure pulses (fast shocks), Geophys. Res. Lett., 26, 1097-1100, 1999.

Zhou, X.-Y. and Tsurutani, B. T.: Interplanetary shock triggering of nightside geomagnetic activity: substorms, pseudobreakups, and quiescent events, J. Geophys. Res., 106, A9, 18 957-18967, 2001. 